\section{Is there a stress component to gastrointestinal disease in cats? A look at the behavioural and environmental risk factors}

\section{Anna Garvey ${ }^{1}$, Aarti Kathrani ${ }^{2}$, Jess Williams ${ }^{1}$, Edward Hall ${ }^{1}$, Emily Blackwell ${ }^{1}$}

1 University of Bristol, Bristol, United Kingdom

2 Royal Veterinary College, London, United Kingdom

\section{OBJECTIVES}

- Calculate the prevalence of gastrointestinal disease in a cohort of domestic pet cats

Identify and quantify, using prospectively collected data, the strength of associations relating to:

- The signalment of the cat

- Diet type

- Exposure to potential environmental stressors

- Personality of the cat that increase/decrease the risk of chronic gastrointestinal disease.

\section{METHODS}

Using data from owner questionnaires, completed as part of the Bristol Cats Study (a longitudinal cohort study of 2444 cats) risk factors for chronic gastrointestinal disease were analysed using multivariate logistic regression. A subset of 40 cats ( 20 cases and 20 unaffected controls) were visited at home. Hair samples were collected for cortisol analysis and behavioural tests were conducted to examine individual differences in response to novelty.

\section{RESULTS}

The prevalence of chronic gastrointestinal disease in the population of cats at 2.5 years old was shown to be $6.8 \%$. Antagonism with other cats in the household, moving house, being indoor only and having cat fights were all associated with an increased odds ratio of having recurrent gastrointestinal disease.

\section{STATEMENT (CONCLUSIONS)}

The risk factors of feline chronic gastrointestinal disease are poorly understood, particularly as to whether there is an affective or stress component to it. These findings demonstrate that daily environmental stressors may have an effect and is therefore an essential finding in promoting the idea that stress should be considered when presented with cats suffering chronic gastrointestinal disease.

\section{Pilot study to} investigate owner education and proficiency in recognising clinical signs of feline osteoarthritis (OA) in UK first opinion practice

\section{Ela Russell ${ }^{1}$, Kelly Bowlt-Blacklock ${ }^{1}$, Russell Chandler ${ }^{2}$}

1 University of Edinburgh, Edinburgh, United Kingdom 2 Alphavet Veterinary Centre, Newport, United Kingdom

\section{OBJECTIVES}

To determine the level of owner education on feline $\mathrm{OA}$

- To measure owner proficiency in recognising the signs of feline $\mathrm{OA}$.

\section{METHODS}

Questionnaires were sent to cat owners registered with one of three UK practices. Information was obtained about cats in the household and owner education about feline OA. Owners were asked whether each of 32 clinical signs were strongly, sometimes or not associated with feline OA. Owner responses were compared using 2-tailed independent t-test.

\section{RESULTS}

Forty-two owners returned the questionnaire. Between $1-10+$ cats lived in each household; respondents owned $1-21+$ cats in their lifetime. 9/42 (21.4\%) of respondent's cats have been treated for $\mathrm{OA}$ and $12 / 42$ (28.6\%) respondents received information from their veterinary practitioner about feline OA, usually as an informal discussion. Mean agreement between owner response and the 